\title{
Segmentação de Núcleos em Imagens Histológicas Renais
}

\author{
Rodrigo E. C. Batista ${ }^{1}$, Rodrigo M. S. Veras ${ }^{1}$, \\ ${ }^{1}$ Universidade Federal do Piauí (UFPI) - Departamento de Computação \\ Teresina - PI - Brasil \\ rodrigoelyelcb@gmail.com, rveras@ufpi.edu.br,
}

\begin{abstract}
This paper proposes a computer-aided diagnosis tool for kidneyrelated pathologies. The proposed system aims to perform the segmentation of cell nuclei in renal biopsy images, serving the diagnosis of several associated pathologies. We performed tests on 122 images treated with Hematoxylin-Eosin (H\&E) and Periodic Acid Schiff (PAS) dyes, sampled from two databases. The method included color deconvolution, morphological, and thresholding operations. A sample of segmented images was evaluated by an expert, who considered $73 \%$ of the segmentations as good or moderate.
\end{abstract}

Resumo. Este trabalho propõe uma ferramenta de diagnóstico auxiliado por computador voltado às patologias relacionadas ao rim. O sistema proposto realiza a segmentação de núcleos celulares em imagens de biópsias renais, servindo ao diagnóstico de diversas patologias associadas. Os testes foram realizados em 122 imagens tratadas com os corantes Hematoxilina-Eosina (H\&E) $e$ Ácido Periódico de Schiff (PAS), amostradas de duas bases de dados. O método incluiu operações de deconvolução de cor, morfológicas e limiarização. Uma amostra das imagens segmentadas foi avaliada por um especialista, que considerou $73 \%$ das segmentações como boas ou regulares.

\section{Introdução}

Cada vez mais a área médica necessita do suporte fornecido pelos sistemas de diagnósticos auxiliado por computação (CAD - Computer Aided Diagnosis). Utilizando esses sistemas o médico pode realizar diagnósticos com mais eficiência e precisão, reduzindo a subjetividade nas suas tarefas. Um diagnóstico sem o auxílio tecnológico inclui tarefas repetitivas e que podem ser comprometidas por condições humanas adversas como estresse e cansaço [Doi 2007].

Uma das vertentes desses sistemas se concentra no processamento de imagens médicas. Nesse sentido o apoio à atividade médica pode ocorrer de diversas maneiras, como efetuar realces, fazer medições de estruturas anatômicas, quantificar características importantes para um diagnóstico ou acompanhar a evolução de doenças por meio de comparações de imagens sequenciais.

Neste trabalho, demos foco para doenças renais. Esse órgão é responsável por funções vitais ao nosso organismo, tais como: eliminação de toxinas do sangue, regulação da formação dos ossos, controle da pressão sanguínea e equilíbrio químico de fluidos do corpo humano.

Quando diagnosticadas tardiamente, ou tratadas de forma inadequada, as doenças renais podem evoluir para estágios avançados de insuficiência renal, condição na qual os 
pacientes devem ser levados ao tratamento de diálise. Segundo a SBN [SBN 2019], cerca de 21 mil pacientes iniciam esse tipo de tratamento no Brasil a cada ano.

Diante da importância de realizar o diagnóstico das doenças renais, neste trabalho nós propomos uma ferramenta de detecção automática dos núcleos celulares, em imagens de biópsias renais. Estruturas essas que são de suma importância para verificar a manifestação da hipercelularidade glomerular, que está presente em diversas doenças [Barros et al. 2017].

Este trabalho está organizado da seguinte forma: na Seção 2 são apresentados os trabalhos relacionados; na Seção 3, são detalhadas as bases de imagens, método proposto e método de avaliação; na Seções 4, aos resultados obtidos e às discussão; por fim, a conclusão e trabalhos futuros são apresentados na Seção 5.

\section{Trabalhos relacionados}

A ferramenta PathoSpotter-K tem como principal objetivo auxiliar patologistas no ensino e em pesquisas sobre doenças renais. Ela se utiliza de aprendizado de máquina e processamento de imagem para identificar automaticamente lesões glomerulares em imagens histológicas de rins, com uma acurácia de 88,3 $\pm 3,6 \%$ [Barros et al. 2017].

Já Tey et. al. [Tey et al. 2017] investigaram dois métodos de detecção glomerular, o método de classificação rLADTree e o método de localização do Espaço de Bowman. Os autores realizaram uma comparação entre estes métodos para analisar o que melhor quantifica as estruturas glomerulares.

No trabalho de Bel et. al. [Bel et al. 2018] foi proposta uma segmentação do tecido renal, utilizando Redes Neurais Convolucionais (CNN). Nos resultados, os autores mostraram que as CNN foram capazes de realizar segmentação com a precisão de $90 \%$ para a diversas estruturas como o glomérulo, túbulo distais, capilares e outros.

Govind et. al. [Govind et al. 2018] desenvolveram um método automatizado para detectar o glomérulo através do filtro Butterworth bandpass filter, que permitiu a extração rápida de características glomerulares de rins. Pode auxiliar em diagnósticos, rastreamento de doenças renais e na progressão da resposta da doença, facilitando na detecção manual dos glomérulos no tecido.

No trabalho de Sheehan e Korstanje [Sheehan and Korstanje 2018], um método semi-automático foi desenvolvido para identificar e coletar informações quantitativas do glomérulo. Os autores usaram realce de contraste e borramento gaussiano, seguido por um filtro para identificar regiões de interesse que correspondem ao glomérulo.

\section{Materiais e Métodos}

Nesta seção dá-se ênfase ao método desenvolvido para segmentar os núcleos em imagens de biópsias renais. Nas subseções a seguir serão detalhadas a base de imagens, o método proposto e o método de avaliação dos resultados.

\subsection{Base de Imagens}

Os testes foram realizados utilizando duas bases de dados, sendo elas: 1) A base do The Jackson Laboratory (TJK) [Sheehan 2018]; 2) Uma base própria obtida em colaboração 
com um Departamento de Medicina Especializada (DME). No geral, foram disponibilizadas 176 imagens com diversos tipos de corantes. Sendo 122 amostras tratadas com Hematoxilina-Eosina (H\&E) e Ácido Periódico de Schiff (PAS) que foram utilizadas neste projeto por conta de serem mais comumente encontradas nas literaturas relacionadas.

Tabela 1. Quantidade de imagens por base.

\begin{tabular}{cccc} 
Corantes & DME & TJK & Total \\
\hline H\&E & 17 & 0 & 17 \\
PAS & 13 & 92 & 105 \\
\hline Total & 30 & 92 & 122 \\
\hline
\end{tabular}

Alguns exemplos utilizados para fazer as segmentações estão demonstrados na Figura 1, onde são representadas por: a) Corante PAS da TJK; b) Corante PAS da DME; c) Corante H\&E da DME.

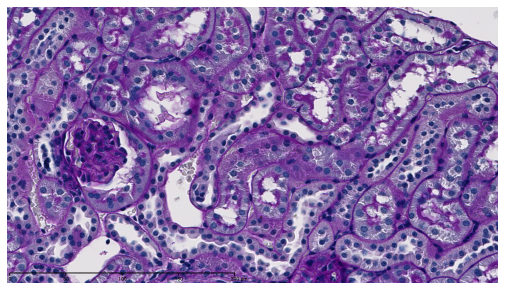

(a)

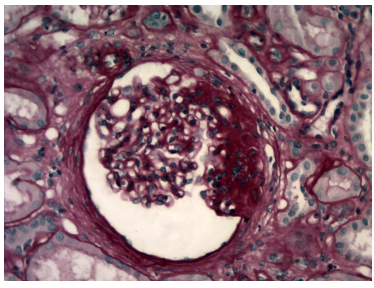

(b)

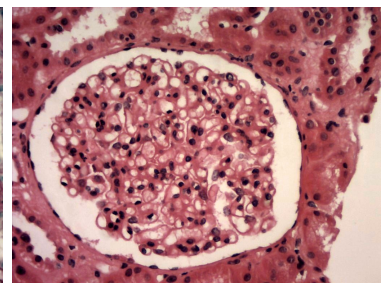

(c)

Figura 1. Amostra de imagens da base de dados: a) Corante PAS - TJK; b) Corante PAS - DME; c) Corante H\&E - DME.

\subsection{Método proposto}

Com esse grande desafio de implementar uma solução para imagens não uniformes, por conta de serem bases com formatos diferentes (dimensões das imagens, corantes iguais porém com tonalidades diferentes e imagens com mais de um glomérulo), surgiu a necessidade de criar diferentes algoritmos para os corantes que estavam em análise. Na implementação foi utilizado a linguagem Python $^{1}$ e a ferramenta Image ${ }^{2}$ em java. A Figura 2 apresenta o passo a passo dos procedimentos utilizados para segmentação das imagens.

Os procedimentos utilizados nas 122 imagens, tiveram como base os procedimento de segmentação da ferramenta PathoSpotter-K [Barros et al. 2017]. Primeiramente foi efetuada uma mudança de espaço de cor para realçar os núcleos nas imagens. Após isso foram aplicadas mudanças de escala de cinza, transformação para negativo e operações morfológicas para que se obtivesse uma visualização clara e unitária de cada núcleo. Em alguns casos, por exemplo: dois núcleos eram considerados como um só, ocorria a exclusão de núcleos muito pequenos e muitos ruídos considerados núcleos. Portanto, foram operações necessárias para que se pudesse obter um resultado bom.

\footnotetext{
${ }^{1}$ https://www.python.org/

${ }^{2}$ https://imagej.nih.gov/ij/
} 


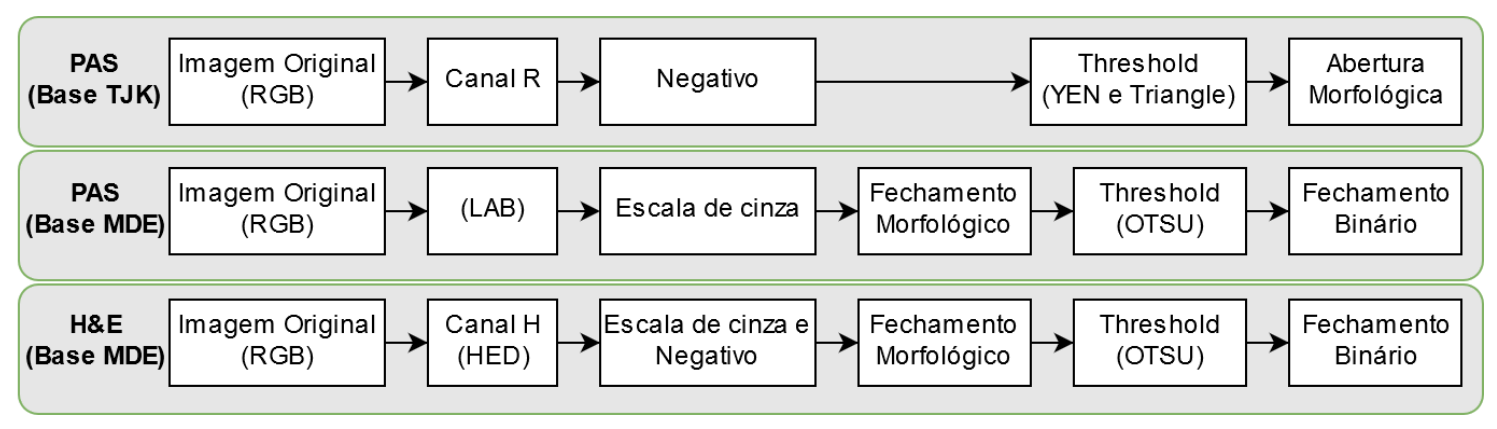

Figura 2. Métodos aplicados sobre as imagens para a segmentação.

Na base TJK com o corante PAS, diferentemente dos outros dois métodos, usamos o canal $\mathrm{R}$ do próprio espaço de cor RGB (canal escolhido de forma empírica para se obter um realce dos núcleos). Logo após, transformamos para o negativo da imagem e realizamos dois procedimentos: dois tipos de limiarização por conta da variedade dos valores dos histogramas, e de forma empírica os threshold (yen e triangle) se encaixaram melhor nos resultados, e foi efetuada uma abertura com o elemento estruturante em forma de disco de tamanho 7.

Nas imagens com corante H\&E da DME, inicia-se alterando o espaço de cor de RGB para o HED [Ruifrok et al. 2001], passando a utilizar o canal "H". Depois transformando em escala de cinza e pegando o negativo da imagem. Após isso aplicamos três procedimentos: um fechamento morfológico, threshold (otsu) e um fechamento binário com o elemento estruturante em forma de quadrado de tamanho 3.

Para o corante PAS, ainda da DME, mudaremos o espaço de cor RGB para LAB, depois transformando a imagem em escala de cinza e novamente realizar três procedimentos: um fechamento morfológico, threshold (otsu) e um fechamento binário com o elemento estruturante em forma de quadrado de tamanho 3.

\subsection{Método de avaliação}

Ao final do processo de segmentação, uma amostra representativa de 37 segmentações, escolhidas aleatoriamente, foram avaliadas por um especialista, que classificou a qualidade do resultado em três categorias: bom, regular ou ruim.

\section{Resultados e discussão}

A aplicação dos procedimentos sobre as imagens geram imagens de mapeamento, (Figura $3 \mathrm{~d}$-f), onde as regiões em branco indicam as respectivas áreas da imagem onde foram detectados os núcleos procurados.

Com as avaliações realizadas pela especialista, produzimos o gráfico presente na Figura 4. Observa-se que dentre as 37 imagens segmentadas, $73 \%$ delas foram considerados boas ou regulares.

Realizamos a análise sobre as imagens segmentadas, e as com H\&E obtiveram melhores avaliações, sendo $88 \%$ consideradas boas, e nenhuma avaliada como ruim (Tabela 2). 


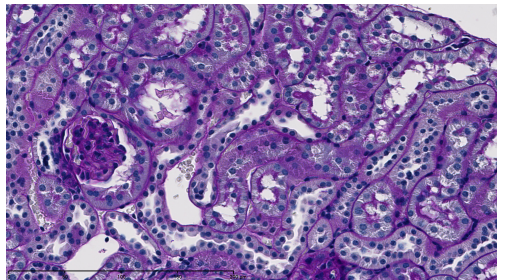

(a)

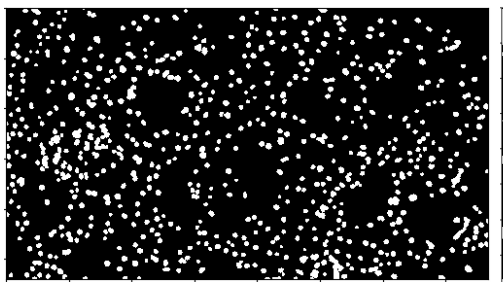

(d)

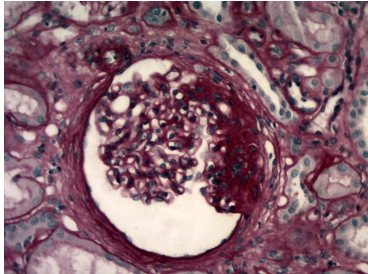

(b)

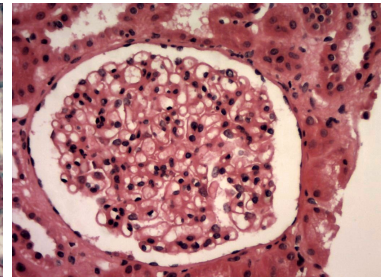

(c)

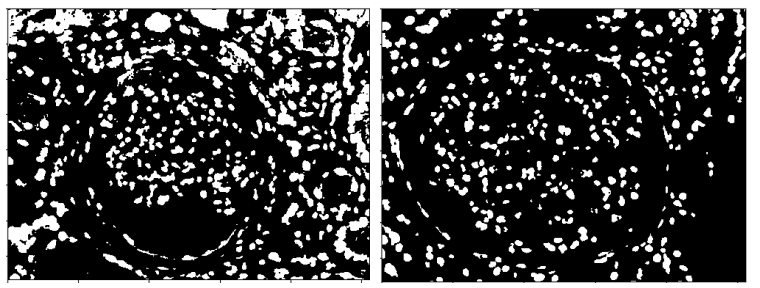

(e)

(f)

Figura 3. Resultados da segmentação, avaliadas como boas. (a) e (d) - PAS base TJK; (b) e (e) - PAS base DME; (c) e (f) - PAS base DME.

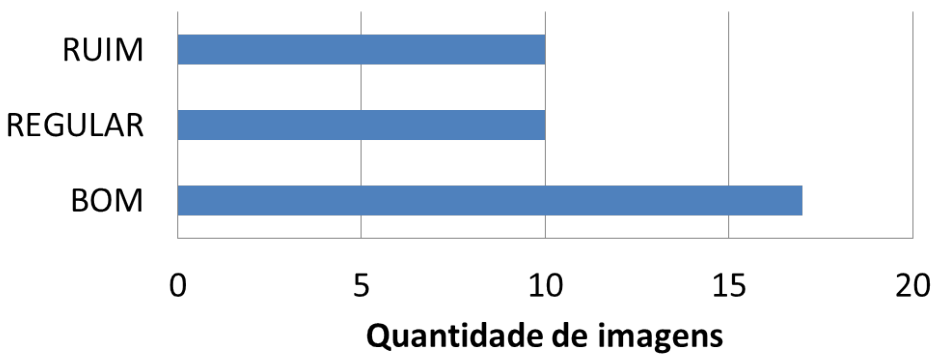

Figura 4. Resultados da avaliação do especialista (ambas as bases).

\section{Conclusão}

É perceptível que atualmente os sistemas de Diagnóstico Auxiliado por Computador possuem um grande potencial e que estão em grande ascensão no mercado global. $O$ fato de diminuir a subjetividade diante de um resultado médico, traz mais precisão e rapidez na descoberta de doenças, que consequentemente, na agilidade do tratamento do mesmo.

Este estudo propôs uma ferramenta que fizesse a segmentação de uma estrutura renal, os núcleos celulares, com o principal intuito de mostrar corretamente as regiões de interesse e ajudar na detecção da hipercelularidade glomerular. Podendo servir diretamente ao médico ou como módulo de outros sistema CAD.

No decorrer do projeto, detectamos a necessidade de indicar ao sistema qual co-

Tabela 2. Tabela de resultados de cada corante e o geral.

\begin{tabular}{c|ccc} 
Corante & Bom & Regular & Ruim \\
\hline H\&E & $88 \%$ & $12 \%$ & $0 \%$ \\
PAS & $35 \%$ & $30 \%$ & $35 \%$ \\
\hline Geral & $46 \%$ & $27 \%$ & $27 \%$ \\
\hline
\end{tabular}


rante está sendo utilizado na imagem. Dada a grande variabilidade de imagens ainda é um desafio fazer um sistema que funcione bem e de maneira genérica.

Diante dos resultados obtidos percebe-se que a separação dos núcleos nas imagens dos corantes H\&E e PAS foram, na maioria, consideradas boas e regulares. Tendo em vista que os resultados bons e regulares foram a maioria, mesmo levando em consideração toda a dificuldade obtida sobre os diferentes tipos de coloração encontradas nas bases de imagens.

Em trabalhos futuros pretendemos fazer melhorias nessa ferramenta, como por exemplo, considerar características de forma e textura das regiões encontradas, para melhorar os resultados.

\section{Referências}

Barros, G. O., Navarro, B., Duarte, A., and Dos-Santos, W. L. (2017). Pathospotter-k: A computational tool for the automatic identification of glomerular lesions in histological images of kidneys. Scientific reports, 7:46769.

Bel, T., Hermsen, M., Smeets, B., Hilbrands, L., van der Laak, J., and Litjens, G. (2018). Automatic segmentation of histopathological slides of renal tissue using deep learning. In Medical Imaging 2018: Digital Pathology, volume 10581, page 1058112. International Society for Optics and Photonics.

Doi, K. (2007). Computer-aided diagnosis in medical imaging: historical review, current status and future potential. Computerized medical imaging and graphics, 31(4-5):198211.

Govind, D., Ginley, B., Lutnick, B., Tomaszewski, J. E., and Sarder, P. (2018). Glomerular detection and segmentation from multimodal microscopy images using a butterworth band-pass filter. In Medical Imaging 2018: Digital Pathology, volume 10581, page 1058114. International Society for Optics and Photonics.

Ruifrok, A. C., Johnston, D. A., et al. (2001). Quantification of histochemical staining by color deconvolution. Analytical and quantitative cytology and histology, 23(4):291299.

SBN (2019). Compreendendo os rins - sociedade brasileira de nefrologia. https://sbn.org.br/publico/institucional/compreendendo-os-rins/. Acesso em: 08/08/2019.

Sheehan, S. M. and Korstanje, R. (2018). Automatic glomerular identification and quantification of histological phenotypes using image analysis and machine learning. American Journal of Physiology-Renal Physiology, 315(6):F1644-F1651.

Tey, W. K., Kuang, Y. C., Khoo, J. J., Ooi, M. P.-L., and Demidenko, S. (2017). Automatic renal interstitial fibrosis quantification system. In 2017 IEEE International Instrumentation and Measurement Technology Conference (I2MTC), pages 1-6. IEEE. 